Documentación de las Ciencias de la Información ISSN-e: $1988-2890$

https://dx.doi.org/10.5209/dcin. 77523

\title{
Verificación del discurso público desde la sociedad civil. Caso de la coalición Ecuador Verifica
}

\author{
Abel Suing ${ }^{1}$; César Ricaurte², Kruzkaya Ordóñez ${ }^{3}$
}

Recibido: 30 de julio de 2021 / Aceptado: 18 de octubre de 2021

Resumen. Reconocer y evitar impactos de las noticias falsas en épocas electorales es ámbito de preocupación para las instituciones, la academia y los ciudadanos porque inciden en la calidad de la democracia, bajo esta premisa se creó la coalición Ecuador Verifica para mitigar el impacto de las redes de desinformación, contribuir con la evaluación de la veracidad del discurso político; y, dotar a la ciudadanía de herramientas para discernir entre información cierta y falsa. Los propósitos del trabajo son compartir el esquema la coalición Ecuador Verifica y evaluar su trabajo. La metodología es cualitativa a través de estudio de caso y entrevistas semiestructuradas. La lucha contra la desinformación es un esfuerzo colectivo, Ecuador Verifica lo aborda desde múltiples perspectivas y gracias a la confluencia de actores de diversa procedencia: medios, sociedad civil y academia, aunque la incorporación de los sectores privado y público ayudaría a la diversidad.

Palabras clave: Fake news; fact-checking; posverdad

\section{[en] Verification of public discourse from civil society. The case of the Ecuador Verifica coalition}

\begin{abstract}
Recognizing and avoiding the impact of fake news in electoral times is an area of concern for institutions, academia and citizens because it affects the quality of democracy. Under this premise, the Ecuador Verifica coalition was created to mitigate the impact of disinformation networks, contribute to the evaluation of the veracity of political discourse, and provide citizens with tools to discern between true and false information. The purposes of the work are to share the Ecuador Verifica coalition scheme and to evaluate its work. The methodology is qualitative through case studies and semi-structured interviews. The fight against disinformation is a collective effort, Ecuador Verifica approaches it from multiple perspectives and thanks to the confluence of actors from diverse backgrounds: media, civil society and academia, although the incorporation of the private and public sectors would help diversity. Keywords: Fake news; fact-checking; post-truth
\end{abstract}

Sumario. 1. Introducción 2. Metodología y objetivos 3. Resultados 5. Conclusiones 5. Bibliografía

Cómo citar: Suing, A.; Ricaurte, C.; Ordóñez, K. (2021). Verificación del discurso público desde la sociedad civil. Caso de la coalición Ecuador Verifica, en Documentación de las Ciencias de la Información. 45 (1), 53-60.

\section{Introducción}

A través de los bulos se edifican anomalías informativas que impactan en la calidad de vida de los ciudadanos porque los alejan de la realidad y afectan sus relaciones sociales, políticas y económicas (Brennen et al., 2020). Los bulos han servido para manipular la opinión pública (Rapp y Salovich, 2018; Valero y Oliveira, 2018; Zhou y Zafarani, 2018) y debilitar el discurso democrático (Codeluppi, 2018; Fisher, 2018), se denominan también noticias falsas o fake news en inglés, irrumpen "en la sociedad en los últimos años y han dado lugar a uno de los momentos más críticos para el periodismo, en el que resulta complicado di- lucidar el grado de veracidad en las informaciones" (Terol-Bolinches y Alonso-López, 2020, p. 306).

La denominación de noticias falsas es un oxímoron, si son noticias falsas no son noticias porque "en el periodismo, en principio, no existe noticia falsa, tanto que una de las reglas es la verificación" (Parreira, 2019, p. 92).

La noticia falsa (distorsionada, engañosa, inconsistente) es una información incorrecta que pretende ser real (Harsin, 2018; Khaldarova y Pantti, 2016; Tandoc et al., 2017), y que circula particularmente en los medios sociales.

El fenómeno de las noticias falsas no es nuevo, surge en panfletos y publicaciones en el siglo XVIII,

\footnotetext{
Universidad Técnica Particular de Loja

E-mail: arsuing@utpl.edu.ec

ORCID: https://orcid.org/0000-0002-4234-5926

2 Fundación Andina para la Observación de Medios, Fundamedios

E-mail: cesar@fundamedios.org

ORCID: https://orcid.org/0000-0002-6819-4388

3 Universidad Técnica Particular de Loja

E-mail: kordonez@utpl.edu.ec

ORCID: https://orcid.org/0000-0002-2360-8188
} 
pero se multiplica masivamente en Internet a través del uso de algoritmos, Big Data e inteligencia artificial (Haigh et al., 2017; Nelson \& Harsh, 2018; Pérez-Dasilva et al., 2020). Se conoce de desinformación durante los grandes conflictos bélicos y entreguerras con la intención de volcar la opinión pública hacia uno de los bandos (Bloch, 1999; Schudson y Zelizer, 2017).

Las noticias falsas se presentan con más frecuencia en decisiones sobre asuntos de interés nacional por ejemplo el acuerdo de paz en Colombia (2016), el referéndum sobre la permanencia del Reino Unido en la Unión Europea (2016), los sufragios en Francia (2017) Italia y México (2018) (Greenberg, 2017; Jankowski, 2018), por ello varias investigaciones destacan el aumento de desinformación en procesos electorales y conflictos (Allcott y Gentzkow, 2017; Pérez-Curiel y García-Gordillo, 2018).

Las redes sociales "juegan un papel fundamental en la producción y difusión de noticias falsas, pero también en su legitimación" (Sued y Rodríguez, 2020, p. 1231), así mismo deforman la libertad de expresión y dan lugar a asociar la posverdad con los ecosistemas digitales (Aparici et al., 2019; Haigh et al., 2017; Pavlik, 2013), en este contexto no es extraño que ayuden a expandir contenidos "cargados de intencionalidad, ya sea política, ideológica o económica" (Mendiguren et al., 2020, p. 173).

Lo contrario de las noticias falsas es el periodismo de verificación, fact-checking en inglés, una práctica que consiste en comprobar a posteriori la información publicada (Mantzarlis, 2018). El fact-checking surge en respuesta a la crisis de confianza de los medios de comunicación, su propósito es disminuir los efectos de las noticias falsas (Gueham, 2017), aplica "técnicas del periodismo de datos para desenmascarar los errores, ambigüedades, mentiras, falta de rigor o inexactitudes de algunos contenidos publicados en los medios de comunicación" (Ufarte-Ruiz et al., 2018, p. 734).

El fact-checking evalúa y califica contenidos de interés y relevancia pública para reducir las percepciones erróneas de los ciudadanos (Nyhan et al., 2019), aumentar la reputación mediática (Nyhan y Reifler, 2014), y dar lugar a una práctica de rendición de cuentas ante la sociedad (Walter et al., 2019).

El éxito del fact-checking "se debe en buena medida a que ha emergido en un contexto de comunicación masiva individualizada, de creciente desconfianza sobre actores que intervienen en el espacio público" (López-García et al., 2016, p. 4). Las iniciativas de periodismo de verificación más reconocidas están relacionadas con política y discurso público (Vázquez-Herrero et al., 2019).

En años recientes surgieron organizaciones de verificación y equipos especializados en los medios de comunicación. En América Latina la mayoría de sistemas de verificación del discurso público se crean fuera de los espacios convencionales y conforman un nuevo ambiente periodístico (Palau-Sampio, 2018; Vizoso y Vázquez-Herrero, 2019).

Concomitantemente al aparecimiento del periodismo de verificación nacen los fact-checkers quienes se encargan de investigar hechos comprobables. Éstos especialistas deben "dominar la comunicación multimedia y en diferentes soportes, debido a que pueden encontrarse con datos e informaciones de diversa naturaleza -vídeo, audio, fotografías, textoque han de saber integrar" (Ufarte-Ruiz, et al., 2020, p. 34), por lo que no resulta exagerado sostener que "los medios de comunicación encuentran en los renovados equipos de fact checkers una vía para mejorar su trabajo y para reforzar los atributos que pueden contribuir a mejorar su imagen y la credibilidad de sus mensajes" (López-García et al., 2016, p. 4).

Las claves para contrarrestar las noticias falsas se encuentran en la deontología, las normas éticas y la concienciación de los periodistas (Blanco-Herrero y Arcila-Calderón, 2019). Lograr cambios será resultado de la colaboración entre "fundaciones, medios de comunicación y academia para establecer un código común que permita un relato coherente y más o menos preciso" (Magallón-Rosa, 2018, p. 280), a partir de lo cual la participación ciudadana contribuya al control de las noticias falsas y a mantener los estándares de calidad de los medios (Lewandowsky et al., 2017). Por lo señalado resulta de interés estudiar las noticias falsas que se publican en los medios de comunicación o circulan en la red (Román-San-Miguel et al., 2020, p. 361).

Para luchar contra la desinformación "numerosas instituciones gubernamentales han publicado páginas especiales que incluyen refutaciones específicas sobre los mitos más recurrentes y perjudiciales" (Pérez-Curiel y Velasco, 2020, p. 90), de igual forma, los medios implementan secciones de verificación abiertas, bajo esta premisa debe mencionarse que la articulación de la sociedad civil con las universidades dio paso a agrupar asociaciones, colectivos y observatorios que luchan contra la desinformación.

La consolidación del fact-checking es evidente en el aparecimiento de las catalogaciones de verificación de noticias como el Duke Reporters' Lab y la Red Internacional de Verificación de Información (IFCN por sus siglas en inglés), además existen indicadores y experiencias constituidas desde sectores privados y empresas de comunicación como el Global Disinformation Index, FactCheck.org, PolitiFact.com y el Washington Post's Fact Checker.

También hay formaciones independientes como Chequeado, Pagella politica y Snopes, financiadas por su propia actividad y otras fundadas por personas sin trayectoria periodística. Chequeado, plataforma argentina, fue la primera experiencia de fact-checking en español. Pagella politica hasta 2016 fue la única organización italiana especializada en verificación de información. Los propósitos de todas son supervisar la exactitud de los hechos y contrastar lo que dicen los principales actores políticos en los 
anuncios televisivos, debates, discursos, entrevistas y comunicados de prensa. Los criterios de verificación son independencia, política abierta, transparencia de fuentes y métodos.

Un proceso de verificación sigue una secuencia, puede ejecutarse en "cuatro fases: identificación y comunicación, discusión, aprobación y publicación, para garantizar la credibilidad. Estos pasos, más las políticas establecidas, garantizan el éxito de los desmentidos" (Bernal-Triviño y Clares-Gavilán, 2019, p. 6), en otros casos hay tres etapas: 1. Búsqueda de declaraciones verificables a través de medios de comunicación y redes sociales, 2. Búsqueda de fuentes originales, 3. Corrección del contenido mostrando las pruebas disponibles (Mantzarlis, 2018), a pesar de esta sistematización los métodos para comprobar datos son insuficientes (Uscinski \& Butler, 2013), se precisa mejorar los "protocolos de comprobación que puedan ayudar a los usuarios a reconocer lo falso" (Pérez-Curiel \& Velasco, 2020, p. 91) y ejecutar herramientas concretas (Amazeen, 2017; Boididou et al., 2018).

Las metodologías de verificación tienen similitud. En Iberoamérica los suscriptores del Código de Principios de la International Fact-Checking Network cumplen un protocolo exhaustivo de informes apartidistas de declaraciones políticas, institucionales o de otros actores sociales, así ha sido con Maldita.es y Newtral (España), Chequeado (Argentina), Colombiacheck y La Silla Vacia (Colombia); y, Ecuador Chequea (Ecuador).

Cada organización o medio establece procedimientos: cuatro etapas (Ecuador Chequea), cinco (Newtral), seis (Colombiacheck y La Silla Vacía), siete (Maldita.es) u ocho (Chequeado), pero la disparidad no implica disminuir la calidad de las examinaciones. Elementos comunes son:

1. Selección de la frase, afirmación o declaración pronunciada en un ámbito público o en redes sociales.

2. Evaluación de relevancia, impacto social y repercusión en el debate público.

3. Consultar las fuentes.

4. Contextualización de la afirmación.

5. Otorgar una calificación sobre la base de categorías establecidas.

A partir de este marco referencial se examina el caso de la coalición Ecuador Verifica una iniciativa inédita en Ecuador que reunió a 18 medios de comunicación, nueve organizaciones de la sociedad civil y siete universidades. Sus objetivos, entre septiembre de 2020 y abril de 2021, fueron la detección de redes que propagan contenido engañoso, la verificación de discurso público, la alfabetización digital como forma de contribuir a la integridad del proceso electoral de presidente y vicepresidente, y desarrollar herramientas que permitan a la ciudadanía decidir sobre la base de información certera. Ecuador Verifica reci- bió apoyo de la cooperación internacional como del Instituto Nacional Demócrata (NDI por sus siglas en inglés) y del Programa de las Naciones Unidas para el Desarrollo (PNUD).

Los objetivos del presente ensayo son compartir esquema de trabajo de Ecuador Verifica y evaluar la experiencia de la coalición en el proceso electoral antes señalado para ubicar elementos que sirvan de insumo para próximas elecciones.

\section{Metodología}

La metodología es cualitativa a través de estudio de caso (Wimmer y Dominick, 1996; Yin, 2009) y entrevistas semiestructuradas. El estudio de caso es pertinente ya que Ecuador Verifica fue la única organización que surgió en Ecuador dedicada a la verificación del discurso de los candidatos. La técnica de estudio de casos permite exponer transformaciones y ofrece recursos al investigador (García-Avilés y González, 2012).

Las entrevistas se realizaron en julio de 2021 en torno a 1) ¿Cuán importante es combatir la desinformación en periodos electorales?, 2) ¿Conoce de Ecuador Verifica y cómo valora su trabajo? La elección de los entrevistados corresponde a una muestra no probabilística por conveniencia debido a la disponibilidad de las personas que participaron en la coalición, este mecanismo optimiza el tiempo y brinda información "de acuerdo con las circunstancias concretas que rodean tanto al investigador como a los sujetos o grupos investigados" (Sandoval, 2002, p. 124). Los perfiles de los entrevistados son un corresponsal internacional, un docente universitario y un presentante de una organización social.

\section{Resultados}

La coalición se gestó debido a que la desinformación tiene un efecto determinante en las agendas informativas, puede marcar el rumbo del proceso electoral, influir en la decisión de los votantes y desestabilizar el entorno político.

Los aliados de Ecuador Verifica contribuyeron a combatir la desinformación y a promover la alfabetización digital. Los contenidos verificados se alojaron en la página www.ecuadorverifica.org además se difundieron en redes sociales y en plataformas. La iniciativa emula otras como Electionland (Estados Unidos), CrossCheck (Francia), Verificado (México), Projeto Comprova (Brasil), y Redcheq (Colombia).

Los medios de comunicación replicaron las notas periodísticas elaboradas por la coalición, compartieron contenidos en sus redes sociales y mantuvieron contacto constante con los voceros de Ecuador Verifica. Las organizaciones brindaron información y apoyo de expertos para verificación y análisis, aler- 
taron sobre la información falsa. Las universidades organizaron debates y talleres de capacitación.

La organización interna de la coalición (tabla 1) orientó la gestión del equipo de redacción central integrado por un editor, cuatro periodistas, un diseñador y un editor de audiencias quienes monitorizaron las principales redes sociales e identificaron conteni- dos engañosos verificables. Se empleó la metodología de fact-checking establecida y avalada por la Red Internacional de Verificación de Información. En armonía con otros casos "el formato de la información a verificar contempló seis tipos: audio, vídeo, imágenes, texto, declaración e información de medios" (Rodríguez-Pérez, 2021, p. 627).

Tabla 1. Organización de Ecuador Verifica

\begin{tabular}{|l|l|l|}
\hline \multicolumn{1}{|c|}{ Áreas } & \multicolumn{1}{|c|}{ Objetivos } & \multicolumn{1}{c|}{ Actividades } \\
\hline $\begin{array}{l}\text { Detección de redes de } \\
\text { desinformación }\end{array}$ & $\begin{array}{l}\text { Mitigar el impacto de las redes } \\
\text { de desinformación en el contexto } \\
\text { electoral 2020-2021. }\end{array}$ & $\begin{array}{l}\text { Detectar contenidos potencialmente falsos } \\
\text { Ubicar el origen de los contenidos poten- } \\
\text { cialmente falsos } \\
\text { Difundir información verificada }\end{array}$ \\
\hline $\begin{array}{l}\text { Verificación del dis- } \\
\text { curso público }\end{array}$ & $\begin{array}{l}\text { Contribuir con la evaluación de } \\
\text { la veracidad del discurso político } \\
\text { mediante la verificación de los } \\
\text { mensajes pronunciados. }\end{array}$ & $\begin{array}{l}\text { Identificar discursos con contenido falso } \\
\text { Verificar la precisión del discurso público } \\
\text { en contexto electoral. } \\
\text { Difundir información verificada }\end{array}$ \\
\hline Alfabetización digital & $\begin{array}{l}\text { Dotar a la ciudadanía ecuatoria- } \\
\text { na de herramientas para discernir } \\
\text { entre información cierta y falsa } \\
\text { durante el proceso electoral. }\end{array}$ & $\begin{array}{l}\text { Ofrecer un espacio para verificar la infor- } \\
\text { mación difundida en redes sociales o me- } \\
\text { dios de comunicación. } \\
\text { Generar y difundir contenido que eduque a } \\
\text { la ciudadanía sobre } \\
\text { la responsabilidad del consumo de informa- } \\
\text { ción. } \\
\text { los procesos electorales. }\end{array}$ \\
\hline
\end{tabular}

Fuente: coalición Ecuador Verifica. Elaboración: propia.

Figura 1. Logotipo

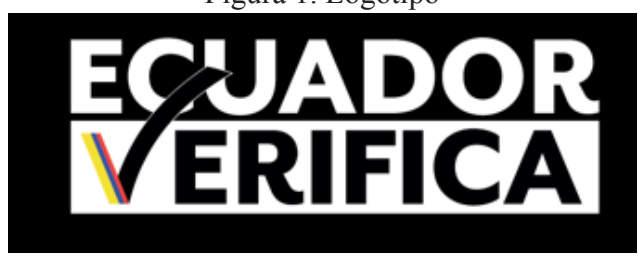

Fuente: coalición Ecuador Verifica. Elaboración: propia.

Las herramientas de verificación empleadas fueron Meltwater, Claim Hunter, Crowdtangle, Fact-Checking tool de Facebook. Se revisó frecuentemente Twitter, Instagram y YouTube, así mismo se acogieron pedidos en WhatsApp. Las verificaciones fueron supervisadas y aprobadas por la dirección de contenidos de la coalición previo a publicarse.

En Ecuador Verifica (figura 2) se revisaron 203 publicaciones que contenían manifestaciones controvertidas. Las categorías para calificar las verificaciones fueron cuatro: 1) cierto, 2) falso, 3) inverificable $\mathrm{y}, 4) \mathrm{si}$, pero. Los contenidos engañosos circularon en Facebook (53\%), Twitter (15\%), WhatsApp (6\%) y Instagram, YouTube y TikTok.

Los temas recurrentes fueron la organización de la primera vuelta electoral, las declaraciones manipuladas de políticos, encuestas y resultados electorales falsos. Los formatos comúnmente usados son montajes, videos editados y suplantación de logotipos.

Figura 2. Contenido en redes sociales

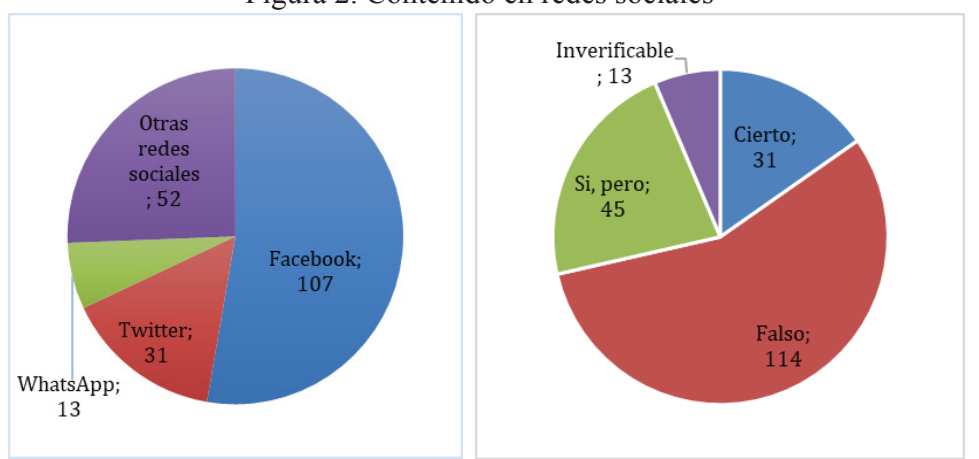

Fuente: coalición Ecuador Verifica. Elaboración: propia. 
Los resultados de la verificación del discurso público de los candidatos a la presidencia y vicepresidencia están en la tabla 2. La mayoría de verificaciones se realizaron durante tres debates presidencia- les de la primera vuelta y uno en la segunda vuelta. Muchas declaraciones de los candidatos carecían de datos o se sustentaron en promesas que no entran en el ámbito de la verificación.

Tabla 2. Verificación del discurso público de los binomios presidenciales

\begin{tabular}{|l|l|l|l|l|l|}
\hline Alianzas / Partidos políticos & Cierto & Falso & Inverificable & Si, pero & Total \\
\hline Creo-Partido Social Cristiano & $6(29 \%)$ & $2(10 \%)$ & $1(5 \%)$ & $12(57 \%)$ & 21 \\
\hline Alianza País & $4(29 \%)$ & $2(14 \%)$ & $1(7 \%)$ & $7(50 \%)$ & 14 \\
\hline $\begin{array}{l}\text { Partido Socialista Ecuatoria- } \\
\text { no-Concertación }\end{array}$ & $2(22 \%)$ & $2(22 \%)$ & $1(11 \%)$ & $4(44 \%)$ & 9 \\
\hline Amigo & $1(20 \%)$ & $3(60 \%)$ & $0(0 \%)$ & $1(20 \%)$ & 5 \\
\hline Avanza & $2(25 \%)$ & $3(38 \%)$ & $1(13 \%)$ & $2(25 \%)$ & 8 \\
\hline Fuerza Ecuador & $1(13 \%)$ & $4(59 \%)$ & $0(0 \%)$ & $3(38 \%)$ & 8 \\
\hline Construye & $0(0 \%)$ & $2(100 \%)$ & $0(0 \%)$ & $0(0 \%)$ & 2 \\
\hline Democracia Sí & $1(20 \%)$ & $3(60 \%)$ & $0(0 \%)$ & $1(20 \%)$ & 5 \\
\hline Izquierda democrática & $6(67 \%)$ & $1(11 \%)$ & $0(0 \%)$ & $2(22 \%)$ & 9 \\
\hline Juntos Podemos & $0(0 \%)$ & $1(50 \%)$ & $0(0 \%)$ & $1(50 \%)$ & 2 \\
\hline Pachakutik & $1(20 \%)$ & $4(80 \%)$ & $0(0 \%)$ & $0(0 \%)$ & 5 \\
\hline Sociedad Patriótica & $2(22 \%)$ & $2(22 \%)$ & $1(11 \%)$ & $4(44 \%)$ & 9 \\
\hline $\begin{array}{l}\text { Sociedad Unida más Acción } \\
\text {-Suma }\end{array}$ & $2(40 \%)$ & $1(20 \%)$ & $0(0 \%)$ & $2(40 \%)$ & 5 \\
\hline UNES & $3(23 \%)$ & $4(31 \%)$ & $0(0 \%)$ & $6(46 \%)$ & 13 \\
\hline Total / Promedios & $25 \%$ & $43 \%$ & $4 \%$ & $35 \%$ & 115 \\
\hline
\end{tabular}

Fuente: coalición Ecuador Verifica. Elaboración: propia.

Desde el área de alfabetización digital se lograron publicar 34 guías para la detección de contenidos desinformativos, conocer sobre derechos digitales y ciberseguridad, otros resultados son la realización de entrevistas a expertos internacionales y cuatro talleres a través de video conferencia impartidos a estudiantes y ciudadanos sobre el uso de herramientas de verificación.

En la página Web de Ecuador Verifica se destinó una sección para notas en quichua que también se difundieron en redes sociales, además se generaron contenidos audiovisuales en este idioma.

Finalmente, se cita la ejecución de los siguientes eventos especiales:

- Ecuador Verifica Live: 24 programas en vivo sobre desinformación electoral y alfabetización digital con entrevistas a expertos nacionales e internacionales.

- Verificación de contenido desinformativo los días de votaciones, y reportes en vivo sobre las jornadas.

- Café de la Verdad: nueve conversatorios informales sobre la labor periodística en época electoral, anécdotas y aprendizajes.

Los impactos de la coalición se evidencian en 102 citas a julio de 2021 en medios de comunicación de Argentina, Australia, Chile, Colombia, Costa
Rica, El Salvador, España, Estados Unidos, Francia, Guatemala, Honduras, México, Nicaragua, Panamá, Paraguay, Suiza, Venezuela. En Facebook y Twitter se recibieron 1778 menciones. Las publicaciones son fuentes de consultas para estudiantes, académicos, organizaciones y medios.

Fruto de las entrevistas debe referirse que la verificación en época electoral "es fundamental como aporte a la democracia, la ciudadanía necesita una guía permanente" (entrevistado 2), "es muy importante, y no solo es responsabilidad del Consejo Nacional Electoral (CNE) sino de los medios de comunicación, de las organizaciones de la sociedad civil, de la ciudadanía y de la academia" (entrevistado 3), pero también debe ocurrir "en otros periodos, es muy importante verificar lo que circula en redes. Con la explosión del Internet es una de las primeras fuentes a través de las cuales la gente se informa" (entrevistado 1).

Respecto a la evaluación de Ecuador Verifica se señaló que "estamos satisfechos con su labor en ambas vueltas. Desde la creación nos pareció completa al tener actores de distintas áreas, desde la academia hasta medios difusores, incluyendo la alfabetización que es clave para que el público tenga herramientas" (entrevistado 1). La coalición "fue necesaria, detalló cómo mejorar. El arranque se hizo con anticipación, se lograron canales de comunicación, de distribución e incluso de estética propia" (entrevistado 3), y "dis- 
paró el interés ciudadano, pero debe sobrepasar el ámbito informativo coyuntural, [por lo que] tiene que trabajar a largo plazo" (entrevistado 2).

\section{Conclusiones}

La lucha contra la desinformación es un esfuerzo colectivo, Ecuador Verifica lo aborda desde múltiples perspectivas y gracias a la confluencia de actores de diversa procedencia: medios, sociedad civil y academia, aunque la incorporación de los sectores privado y público ayudaría a la diversidad.

La coalición inició con un llamado abierto, luego cursó invitaciones a los representantes de las asociaciones productivas e instituciones de todas las funciones del Estado. Hasta octubre de 2021, cuando arranca una segunda etapa, se han sumado otros medios de comunicación y se presentaron las conclusiones del proceso de verificación en las elecciones presidenciales al $\mathrm{CNE}$, siendo bien acogidas en el encuentro "Pandemia, desinformación y elecciones", parte del proyecto "Apoyo al Ciclo Electoral en Ecuador 2020-2022", que es una iniciativa del CNE y el Tribunal Contencioso Electoral, con el apoyo del Programa de las Naciones Unidas en su implementación y la financiación de la Unión Europea, la Agencia Española de Cooperación Internacional para el Desarrollo y la Embajada de Suiza (EFE, 2021; Suing, 2021).

La convocatoria de Ecuador Verifica se sustenta en trabajo colaborativo, apoyos financieros y operati- vos de las partes y fundamentalmente en compromisos deontológicos que ayuden a encontrar elementos claves para la vida democrática del país, no supone coerción ni repuestas a ideologías, su ideario está cercano a las propuestas de filósofos como Adela Cortina y Jesús Conill (2017) respecto a los valores de la ética cívica: libertad, igualdad, solidaridad, respecto activo y diálogo.

Las verificaciones se respaldan en metodologías testeadas, con rigor e independencia editorial. Se logró resultados de verificación en redes sociales (figura 2) y del discurso público (tabla 2), pero debe desarrollarse un programa plural e inclusivo de alfabetización mediática bajo la perspectiva de aportar competencias para que los ciudadanos estén conscientes de sus derechos y deberes en el espacio digital.

Entre los horizontes del colectivo están fomentar relaciones en la región, establecer diálogos y mesas de trabajo con los sectores no incorporados aún, fortalecer las capacidades técnicas y multiplicar el impacto apoyándose en un programa de comunicación estratégica.

Un reto de los ciudadanos es incidir en los partidos políticos y sus candidatos para que cultiven discursos fundados en la verdad, que sus liderazgos se construyan a partir de criterios sustentados. Los datos de la figura 2 y la tabla 2 muestran que más del $40 \%$ de las expresiones sujetas a verificación fueron falsas, y menos de la cuarta parte reales. Queda una línea para comparar en próximas elecciones del país andino.

\section{Bibliografía}

Allcott, H. y Gentzkow, M. (2017). Social media and fake news in the 2016 election. Journal of Economic Perspectives, 31(2), 211-236. https://doi.org/10.1257/jep.31.2.211.

Amazeen, A. (2017). Journalistic interventions: The structural factors affecting the global emergence of fact-checking. Journalism, Sept. 8th. https://doi.org/10.1177/1464884917730217.

Aparici, R., García-Marín, D. y Rincón-Manzano, L. (2019). Noticias falsas, bulos y trending topics. Anatomía y estrategias de la desinformación en el conflicto catalán. El profesional de la información, 28(3). https://doi.org/10.3145/ epi.2019.may.13.

Bernal-Triviño, A. \& Clares-Gavilán, J. (2019). Uso del móvil y las redes sociales como canales de verificación de fake news. El caso de Maldita.es. El profesional de la información, 28(3), 1-8. https://doi.org/10.3145/epi.2019.may.12.

Blanco-Herrero, D. y Arcila-Calderón, C. (2019). Deontología y noticias falsas: estudio de las percepciones de periodistas españoles. El profesional de la información, 28(3). https://doi.org/10.3145/epi.2019.may.08.

Bloch, Marc (1999). Historia e historiadores. Akal.

Boididou, C., Middleton. S., Zhiwei, J., Papadopoulos, S., Dang-Nguyen, D., Boato, G. y Kompatsiaris, Y. (2018). Verifying information with multimedia content on Twitter. Multimedia tools and applications, 77(12), 15545-15571. https://doi.org/10.1007/s11042-017-5132-9.

Brennen, S., Simon, M., Howard, N. y Nielsen, K. (2020). Types, sources, and claims of COVID-19 misinformation. Reuters Institute. https://reutersinstitute.politics.ox.ac.uk/types-sources-and-claims-covid-19-misinformation.

Codeluppi, V (2018). Il tramonto della realtà. Come i media trasformano le nostre vite. Carocci.

Conill, J. (2017). Nuevos horizontes de economía ética en tiempo de neurociencia. Pensamiento, 73 (276), $541-562$.

EFE (18 de agosto de 2021). Ecuador, Colombia, Bolivia y Perú firman declaración para combatir la desinformación electoral. El Universo. https://www.eluniverso.com/noticias/internacional/ecuador-colombia-bolivia-y-peru-firman-declaracion-para-combatir-la-desinformacion-electoral-nota/

Fisher C. (2018) What Is Meant By ‘Trust' In News Media? En K. Otto y A. Köhler (Eds.) Trust in Media and Journalism. Springer VS, Wiesbaden. https://doi.org/10.1007/978-3-658-20765-6_2 
García-Avilés, J. y González, L. (2012). Cibermedios nativos españoles: explorando modelos de rentabilidad. Trípodos, 30, 153-167.

Greenberg, D. (2017). Deciding What's True: The Rise of Political Fact-Checking in American Journalism. Journal of Communication, 67(6), E1-E3. https://doi.org/10.1111/jcom.12329.

Gueham, F. (2017). Le fact-checking: une réponse à la crise de l'information et de la démocratie. Fondation pour l'innovation politique. http:/www.fondapol.org/etude/farid-gueham-le-fact-checking-une-reponse-a-la-crise-de-linformation-et-de-la-democratie.

Haigh, M., Haigh, T. y Kozak, I. (2017). Stopping Fake News. The work practices of peer-to-peer counter propaganda. Journalism Studies, 19(14), 2062-2087. https://doi.org/10.1080/1461670X.2017.1316681.

Haigh, M., Haigh, T. \& Kozak, I. (2017). Stopping fake news. The work practices of peer-to-peer counter propaganda. Journalism Studies, 25, 1-26 https://doi.org/10.1080/1461670X.2017.1316681.

Harsin, J. (2018). A critical guide to fake news: From comedy to tragedy. Pouvoirs. Revue française d'études constitutionnelles et politiques, (164), 99-119.

Jankowski, W. (2018). Researching Fake News: A Selective Examination of Empirical Studies. Journal of the European Institute for Communication and Culture, 25(1-2), 248-255. https://doi.org/10.1080/13183222.2018.1418964.

Khaldarova, I. y Pantti, M. (2016). Fake news. The narrative battle over the Ukrainian conflict. Journalism practice, 10(7), 891-901. https://doi.org/10.1080/17512786.2016.1163237

Lewandowsky, S., Ecker, K. y Cook, J. (2017). Beyond Misinformation: Understanding and Coping with the "Post-Truth" Era. Journal of Applied Research in Memory and Cognition, 6(4), 353-369. https://doi.org/10.1016/j.jarmac.2017.07.008.

López-García, X., Rodríguez-Vázquez, A-I. y Álvarez-Gromaz, L. (2016). El fact checking como reclamo y como servicio en los cibermedios. Análisis de las experiencias de 'The Washington Post' y 'The Guardian'. Telos, 13. https:// telos.fundaciontelefonica.com/archivo/numero103/analisis-de-las-experiencias-de-the-washington-post-y-the-guardian/?output=pdf.

Magallón-Rosa, R. (2018). La biblioteca digital sobre Donald Trump. Fact-checking frente a fake news. Estudios sobre el mensaje periodístico, 24(1), 273-282. https://doi.org/10.5209/ESMP.59949.

Mantzarlis, A. (2018). Fact-checking 101. En C. Ireton y J. Posetti (Eds.), Journalism, fake news \& disinformation: Handbook for journalism education and training (85-100). Unesco. https://unesdoc.unesco.org/ark:/48223/pf0000265552.

Mendiguren, T., Pérez, J., y Meso, K. (2020). Actitud ante las Fake News: Estudio del caso de los estudiantes de la Universidad del País Vasco. Revista de Comunicación, 19(1), 171-184. https://doi.org/10.26441/RC19.1-2020-A10.

Nelson, L. y Harsh, T. (2018). The small, disloyal fake news audience: The role of audience availability in fake news consumption. Communication research, 18. https://doi.org/10.1177/1461444818758715.

Nyhan, B. y Reifler, J. (2014). The effect of fact-checking on elites: A field experiment on U.S. state legislators. American Journal of Political Science, 59(3), 628-640. http://dx.doi.org/10.1111/ajps.12162.

Nyhan, B., Porter, E., Reifler, J., y Wood, T. (2019). Taking fact-checks literally but not seriously? The effects of journalistic fact-checking on factual beliefs and candidate favorability. Political Behavior, 1-22. https://doi.org/10.1007/ s11109-019-09528-x.

Palau-Sampio, D. (2018). Fact-checking y vigilancia del poder: La verificación del discurso público en los nuevos medios de américa latina. Communication \& Society, 31(3), 347-365. https://doi.org/10.15581/003.31.3.347-365.

Parreira, M. (2019). La proliferación de las "fake news" y sus algoritmos daña la cultura democrática. Ámbitos. Revista Internacional de Comunicación, 45, 89-106.

Pavlik, J. (2000). The impact of technology on journalism. Journalism studies, 1(2), 229-237. https://doi. org/10.1080/14616700050028226.

Pérez-Curiel, C. y Velasco, A. M. (2020). Impacto del discurso político en la difusión de bulos sobre Covid-19. Influencia de la desinformación en públicos y medios. Revista Latina de Comunicación Social, 78, 86-118. https://doi. org/10.4185/RLCS-2020-1469.

Pérez-Curiel, C. y García-Gordillo, M. (2018). Política de influencia y tendencia fake en Twitter. Efectos postelectorales (21D) en el marco del Procés en Cataluña. El profesional de la información, 27(5), 1030-1040. https://doi. org/10.3145/epi.2018.sep.07.

Pérez-Dasilva, J. Á.; Meso-Ayerdi, K. y Mendiguren-Galdospín, T. (2020). Fake news y coronavirus: detección de los principales actores y tendencias a través del análisis de las conversaciones en Twitter. El profesional de la información, 29(3), e290308. https://doi.org/10.3145/epi.2020.may.08.

Rapp, N. y Salovich, A. (2018). Can't We Just Disregard Fake News? The Consequences of Exposure to Inaccurate Information. PolicyInsights from the Behavioral and Brain Sciences, 5(2), 232-239. https://doi.org/10.1177/2372732218785193.

Rodríguez-Pérez, C. (2021). Desinformacion online y fact-checking en entornos de polarización social: el periodismo de verificación de Colombiacheck, La Silla Vacía y AFP durante la huelga nacional del $21 \mathrm{~N}$ en Colombia. Estudios sobre el mensaje Periodístico, 27(2), 623. https://doi.org/10.5209/esmp.68433.

Román-San-Miguel, A., Sánchez-Gey, N. y Elías-Zambrano, R. (2020). Las fake news durante el Estado de Alarma por COVID-19. Análisis desde el punto de vista político en la prensa española. Revista Latina de Comunicación Social, 78, 359-391. https://doi.org/10.4185/RLCS-2020-1481. 
Sandoval, C. (2002). Investigación Cualitativa. Programa de especialización en teoría, métodos y técnicas de investigación social. Arfo Editores.

Schudson, M. y Zelizer, B. (2017). Fake News in context. En VV. AA., Understanding and Addressing the Disinformation Ecosystem (pp. 1-4). Annenberg School for Communication.

Sued, G. y Rodríguez, M. (2020): Noticias falsas en Facebook: narrativas, circulación y verificación. Los casos de Argentina y México. Estudios sobre el mensaje periodístico 26(3), 1229-1242.

Suing, A. (17 de agosto de 2021). Alfabetización informacional y mediática para la formación ciudadana y toma de decisiones informadas en democracia. Experiencia y aporte desde UTPL, Ecuador. Zenodo. https://doi.org/10.5281/ zenodo.5237476.

Tandoc, E., Lim, Z-W y Ling, R. (2017) “Defining “fake news”: A typology of scholarly definitions”. Digital journalism, $6(2), 137-153$.

Terol-Bolinches, R., \& Alonso-López, N. (2020). La Prensa Española en la era de la posverdad: el compromiso de la verificación de datos para combatir las fake news. Revista Prisma Social, 31, 304-327.

Ufarte-Ruiz, J., Anzera, G. y Murcia-Verdú, J. (2020). Plataformas independientes de fact-checking en España e Italia. Características, organización y método. Revista Mediterránea de Comunicación, 11(2), 23-39. https://www.doi. org/10.14198/MEDCOM2020.11.2.3.

Ufarte-Ruiz, M-J., Peralta-García, L. y Murcia-Verdú, F-J. (2018). "Fact checking: un nuevo desafío del periodismo". El profesional de la información, 27(4), 733-741. https://doi.org/10.3145/epi.2018.jul.02.

Uscinski, J. y Butler, W. (2013). "The epistemology of fact checking”. Critical review. A journal of politics and society, 25(2), 162-180. https://doi.org/10.1080/08913811.2013.843872.

Valero, P., y Oliveira, L. (2018). Fake news: una revisión sistemática de la literatura. Observatorio (OBS*), 12(5). https:// doi.org/10.15847/obsOBS12520181374.

Vázquez-Herrero, J., Vizoso, A. \& López-García, X. (2019). Innovación tecnológica y comunicativa para combatir la desinformación: 135 experiencias para un cambio de rumbo. Profesional de la información, 28(3). https://doi. org/10.3145/epi.2019.may.01

Vizoso, Á, \& Vázquez-Herrero, J. (2019). Plataformas de fact-checking en español. características, organización y método. Communication \& Society, 32(1), 127-144. https://doi. org/10.15581/003.32.1.127-144

Walter, N., Cohen, J., Holbert, L., \& Morag, Y. (2019). Fact-checking: A meta-analysis of what works and for whom. Political Communication, 1-26. http://dx.doi.org/10.1080/10584609.2019.1668894

Wimmer, R. \& Dominick, J. (1996). La investigación cientifica de los medios de comunicación. Bosch Comunicación.

Yin, K. (2009). Case study research. Design and methods. Thousand Oaks: Sage.

Zhou, X. \& Zafarani, R. (2018). Fake news: A survey of research, detection methods, and opportunities. ACM Computing Surveys, 1, 1-40. DOI 10.1145/3395046 